DIRECT TRANSISTOR-LEVEL LAYOUT FOR DIGITAL BLOCKS 


\section{DIRECT TRANSISTOR-LEVEL LAYOUT FOR DIGITAL BLOCKS}

PRAKASH GOPALAKRISHNAN

Neolinear, Inc.

ROB A. RUTENBAR

Carnegie Mellon University

\section{KLUWER ACADEMIC PUBLISHERS}

NEW YORK, BOSTON, DORDRECHT, LONDON, MOSCOW 
eBook ISBN: $\quad 1-4020-8063-8$

Print ISBN: $\quad 1-4020-7665-7$

C2005 Springer Science + Business Media, Inc.

Print C2004 Kluwer Academic Publishers

Boston

All rights reserved

No part of this eBook may be reproduced or transmitted in any form or by any means, electronic, mechanical, recording, or otherwise, without written consent from the Publisher

Created in the United States of America

Visit Springer's eBookstore at:

http://ebooks.kluweronline.com

and the Springer Global Website Online at:

http://www.springeronline.com 


\section{Table of Contents}

Table of Contents

$\begin{array}{ll}\text { Preface } & \text { ix }\end{array}$

Chapter 1 Introduction 1

1.1 Motivation: Standard Cells vs. Transistors ................................. 1

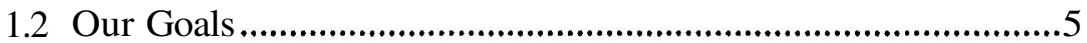

1.3 Previous Transistor-Level Approaches ......................................6

1.4 Overall Strategy ..................................................................... 9

1.5 Book Outline................................................................................. 12

Chapter 2 Circuit Structure And Clustering 13

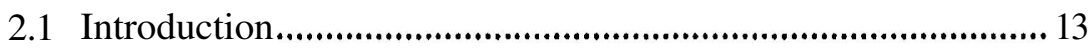

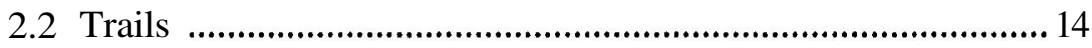

2.3 Essential Clusters and Circuit Structure .................................. 17

2.4 Pattern Matching ................................................................20

2.5 Circuit Structure Library ......................................................22

2.6 Benchmarks, Clustering Experiments and Results ....................24 
2.6.1Benchmarks for Experiments .............................................24

2.6.2Synthesis Target Library Comparison ..............................26

2.6.3Circuit Structure Library Example......................................33

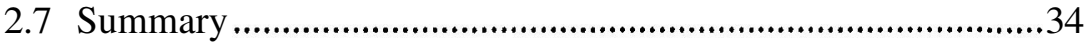

Chapter 3 Global Placement $\quad 35$

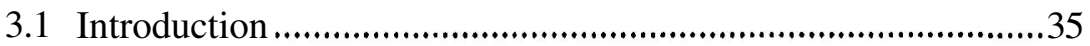

3.2 Quadratic Placement and Partitioning .....................................38

3.2.1Quadratic Solve .................................................................38

3.2.2Recursive Re-Partitioning ...............................................40

3.2.3Bi-Partitioning Improvement ........................................40

3.3 Simulated Annealing Legalization............................................41

3.4 Global Placement Results ......................................................4

3.4.1Congestion during Global Placement .............................45

3.4.2Row Utilization and Congestion......................................45

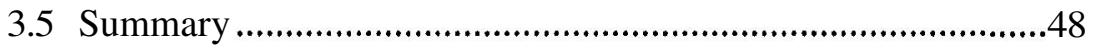

\section{Chapter 4 Detailed Placement And Layout Results 49}

4.1 Introduction ..........................................................................49

4.2 Intra-Cluster Optimizations ....................................................51

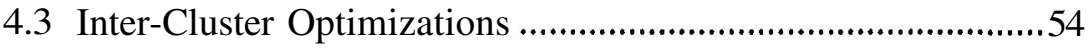

4.4 Global Routability............................................................56

4.5 Local Placement Optimization..............................................56

4.5.1Pair-Wise Cluster Merges .................................................56

4.5.2Greedy Local Optimization ..........................................64

4.5.3Dynamic Programming Local Optimization....................64

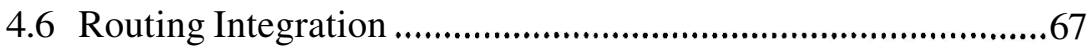

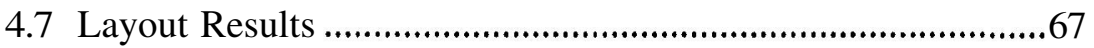

4.7.1Simple Layout Example..............................................67

4.7.2Layout Comparison Experiments .....................................72

4.7.3Dynamic Programming vs. Greedy Local Optimization .74 


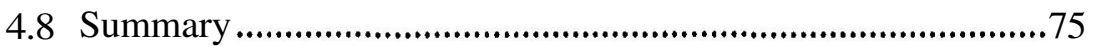

$\begin{array}{lll}\text { Chapter } 5 & \text { Timing-Driven Placement } & 77\end{array}$

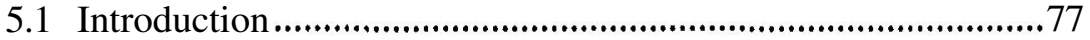

5.2 Transistor-Level Timing Analysis ............................................78

5.2.1DCCs: DC-Coupled Components.....................................79

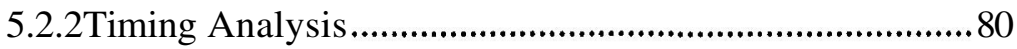

5.2.3Transistor-Level Static Timing ........................................81

5.3 Delay Graphs and Critical Paths ...........................................85

5.4 Timing-Driven Global Placement ............................................ 88

5.4.1Timing-Driven Placement Techniques..............................8 89

5.4.2Net-weights from Critical Paths .........................................99

5.4.3Net-Weight / Timing-Driven Placement ...........................91

5.4.4Interconnect Delays ............................................................991

5.4.5DCC Delay Macro-Modeling .........................................92

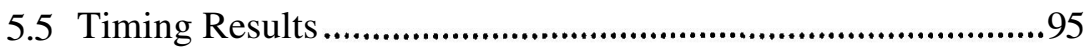

5.5.1Delay Improvement Results............................................96

5.5.2Netlist Structure and Path Distribution..............................97

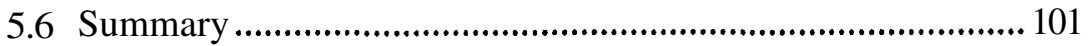

$\begin{array}{lll}\text { Chapter } 6 & \text { Conclusion } & 103\end{array}$

$\begin{array}{ll}\text { Appendix } & 107\end{array}$

$\begin{array}{ll}\text { Bibliography } & 115\end{array}$

$\begin{array}{ll}\text { Index } & 123\end{array}$ 


\section{Preface}

Cell-based design methodologies have dominated layout generation of digital circuits. Unfortunately, the growing demands for transparent process portability, increased performance, and low-level device sizing for timing/power are poorly handled in a fixed cell library. This motivated our search for an alternative layout technique, which has resulted in the direct transistor-level layout approach that we describe in this book. This new approach better accommodates demands for devicelevel flexibility for small blocks of custom digital logic. It captures essential shape-level optimizations, yet scales easily to netlists with thousands of devices, and incorporates timing optimization during layout.

This book would not have been possible without the support from several of our colleagues, friends and family. While we cannot mention all of them, we are particularly grateful to our spouses Surya Viswanathan, and Martha Baron; Prof. Larry Pileggi \& Prof. Rick Carley of Carnegie Mellon University and Dr. Jeff Burns of IBM for their guidance and feedback throughout the research work; John Cohn \& Dave Hathaway of IBM and Bill Halpin \& Artour Levin of Intel for valuable discussions; Prof. Herman Schmit, Vikas Chandra, Aneesh Koorapaty, Emrah Acar, Ravishankar Arunachalam and Anoop Iyer for help with various experiments; colleagues and friends from Neolinear for their understanding and support. Finally, we thank the Semiconductor Research Corporation (SRC) for having provided the financial support for this work.

PRAKASH GOPALAKRISHNAN

NEOLINEAR, INC.

ROB A. RUTENBAR

CARNEGIE MELLON UNIVERSITY 